\title{
STEINER SYMMETRY IN THE MINIMIZATION OF THE FIRST EIGENVALUE IN PROBLEMS INVOLVING THE $p$-LAPLACIAN
}

\author{
CLAUDIA ANEDDA AND FABRIZIO CUCCU
}

(Communicated by Joachim Krieger)

\begin{abstract}
Let $\Omega \subset \mathbb{R}^{N}$ be an open bounded connected set. We consider the eigenvalue problem $-\Delta_{p} u=\lambda \rho|u|^{p-2} u$ in $\Omega$ with homogeneous Dirichlet boundary condition, where $\Delta_{p}$ is the $p$-Laplacian operator and $\rho$ is an arbitrary function that takes only two given values $0<\alpha<\beta$ and that is subject to the constraint $\int_{\Omega} \rho d x=\alpha \gamma+\beta(|\Omega|-\gamma)$ for a fixed $0<\gamma<|\Omega|$. The optimization of the map $\rho \mapsto \lambda_{1}(\rho)$, where $\lambda_{1}$ is the first eigenvalue, has been studied by Cuccu, Emamizadeh and Porru. In this paper we consider a Steiner symmetric domain $\Omega$ and we show that the minimizers inherit the same symmetry.
\end{abstract}

\section{INTRODUCTION}

In this paper we consider the eigenvalue problem

$$
\begin{cases}-\Delta_{p} u=\lambda \rho|u|^{p-2} u & \text { in } \Omega, \\ u=0 & \text { on } \partial \Omega,\end{cases}
$$

where $\Delta_{p}$ denotes the $p$-Laplace operator, $\Delta_{p} u=\operatorname{div}\left(|\nabla u|^{p-2} \nabla u\right), p>1, \Omega \subset \mathbb{R}^{N}$ $(N \geq 2)$ is a bounded domain with $C^{2, \sigma}$ boundary $\partial \Omega, \rho \in L^{\infty}(\Omega)$ is positive and $\lambda \in \mathbb{R} ; u \in W_{0}^{1, p}(\Omega)$ is a weak solution of (1.1) if

$$
\int_{\Omega}|\nabla u|^{p-2} \nabla u \cdot \nabla \xi d x=\lambda \int_{\Omega} \rho|u|^{p-2} u \xi d x \quad \forall \xi \in C_{0}^{\infty}(\Omega) .
$$

A nontrivial solution of (1.1) is called an eigenfunction associated to the eigenvalue $\lambda$; the least real number $\lambda$ with the previous property is said to be the first eigenvalue and every associated eigenfunction is called a first eigenfunction.

The case $\rho \equiv 1$ has been studied by various authors. As observed in [20, the existence of a nontrivial solution of (1.1) follows from direct methods of calculus of variations. In 1986, de Thélin [11] proved that the first eigenvalue is simple when $\Omega$ is the unit ball. In 1987, Sakaguchi [20] proved the simplicity when $\partial \Omega$ is smooth and connected. In 1988, Barles [2] extended the last result when $\partial \Omega$ is of class $C^{2, \sigma}$. In 1990, Lindqvist [16] showed the simplicity of the first eigenvalue in any bounded domain.

In 1987, under the assumption $\partial \Omega \in C^{2, \sigma}$, Anane [1] proved that the first eigenvalue is simple and isolated for an arbitrary $\rho \in L^{\infty}(\Omega)$ such that the set $\{x \in \Omega: \rho(x)>0\}$ has positive Lebesgue measure.

Received by the editors September 7, 2015 and, in revised form, September 30, 2015.

2010 Mathematics Subject Classification. Primary 35J20, 35P15, 47A75.

Key words and phrases. Eigenvalue problem, minimization, Steiner symmetry. 
Physically, in the case of $p=2$, problem (1.1) models a vibrating membrane $\Omega$ with clamped boundary $\partial \Omega$ and density $\rho$. The physical meaning of the first eigenvalue $\lambda_{1}$ is the principal natural frequency of vibration of the membrane.

Note that densities that differ from each other by a subset of Lebesgue null measure give the same eigenvalue problem.

Fixing the positive constants $\alpha, \beta, \gamma$ with $\alpha<\beta$, we restrict our attention to eigenvalue problems where the weight is of the form $\rho=\alpha \chi_{E}+\beta\left(1-\chi_{E}\right)$, where $\chi_{E}$ is the characteristic function of an arbitrary measurable set $E \subset \Omega$ such that $|E|=\gamma$, where $|E|$ denotes the measure of $E$. As $E$ varies, it describes the set we call $a d_{\gamma}$. We denote by $\lambda_{1}(\rho)$ the dependence of the first eigenvalue on the weight $\rho$.

The problem of minimization of $\lambda_{1}(\rho)$ when $\rho$ varies in $a d_{\gamma}$ has been studied in [9] (in the case of an arbitrary positive function $\rho \in L^{\infty}(\Omega)$ ). The authors proved the existence and a characterization of minimizers. Call $\check{\rho}$ such a minimizer. In this paper, we investigate the geometric properties of minimizers when $\Omega$ is a Steiner symmetric domain. A similar problem in the case $p=2$ has been already studied in [4] by Chanillo and others; in that paper the authors proved the Steiner symmetry of every minimizer when $\Omega$ has such a symmetry. In [19], Pielichowski considered the optimization problem in [4] for an arbitrary $p>1$.

To our knowledge, until now for arbitrary $p$ only a weaker symmetry result has been shown, i.e., if $\Omega$ is a ball, then every minimizer is radially symmetric and decreasing (see [9] and [19]). In this paper, we prove the Steiner symmetry of minimizers provided $\Omega$ is Steiner symmetric.

Similar problems for $p=2$ are considered in [5], 6], 8, 13] and [14.

This article is organized in this way: in Section 2 we give some notation and preliminary tools that will be used in the sequel; Section 3 contains the symmetry result.

\section{Preliminaries And notation}

First, we list some regularity properties of the eigenfunctions. Let $u$ be an eigenfunction of (1.1). By Propositions 2.1 and Remark 2.3 in [10] we know that

$$
u \in W_{0}^{1, p}(\Omega) \cap C_{\mathrm{loc}}^{1, \tau}(\Omega) \cap L^{\infty}(\Omega) .
$$

Moreover by results of Tolksdorf in 22, $u$ has weak second derivatives; more precisely,

$$
\begin{gathered}
u \in W_{\mathrm{loc}}^{2,2}(\Omega) \cap W_{\mathrm{loc}}^{1, \infty}(\Omega) \quad \text { if } p \geq 2, \\
u \in W_{\mathrm{loc}}^{2, p}(\Omega) \cap W_{\mathrm{loc}}^{1, p+2}(\Omega) \quad \text { if } p \leq 2 .
\end{gathered}
$$

Furthermore it has been shown in [1] that $\lambda_{1}(\rho)$ is simple and every first eigenfunction $u$ is either positive or negative in $\Omega$.

We will use the following variational characterization of the first eigenvalue $\lambda_{1}(\rho)$ of (1.1):

$$
\lambda_{1}(\rho)=\min _{\substack{u \in W_{0}^{1, p}(\Omega) \\ u \neq 0}} \frac{\int_{\Omega}|\nabla u|^{p} d x}{\int_{\Omega} \rho u^{p} d x}
$$

the expression $\frac{\int_{\Omega}|\nabla u|^{p} d x}{\int_{\Omega} \rho u^{p} d x}$ is the well-known Rayleigh's quotient. 
Let $a d_{\gamma}=\left\{\rho \in L^{\infty}(\Omega): \rho=\alpha \chi_{E}+\beta\left(1-\chi_{E}\right)\right\}$, where $0<\alpha<\beta$ and $\chi_{E}$ is the characteristic function of a set $E \subset \Omega$ such that $|E|=\gamma$. We denote by $\check{\rho} \in a d_{\gamma}$ a minimizer of the map $\rho \mapsto \lambda_{1}(\rho)$, that is,

$$
\lambda_{1}(\check{\rho})=\inf _{\rho \in a d_{\gamma}} \lambda_{1}(\rho) .
$$

From results in 9] such a minimizer exists; in the sequel we will write $\check{\lambda}_{1}=\lambda_{1}(\check{\rho})$. Moreover (see [9]), if $u$ is a positive eigenfunction corresponding to $\lambda_{1}(\rho)$, then there exists an increasing function $\varphi$ such that

$$
\check{\rho}=\varphi(u)
$$

almost everywhere in $\Omega$.

Since in our particular case $\varphi$ takes only the values $\alpha$ and $\beta$, characterization (2.3) can be stated more precisely as the following.

Proposition 2.1. Let $u$ be a positive first eigenfunction of problem (1.1) with $\rho(x)=\check{\rho}(x)$; then there exists a constant $l>0$ such that

$$
\check{\rho}(x)= \begin{cases}\beta & \text { if } u(x)>l, \\ \alpha & \text { if } u(x) \leq l,\end{cases}
$$

for every $x \in \Omega$.

Now we introduce some notation and recall the notion of Steiner symmetric set and function. Throughout the paper, a point $x \in \mathbb{R}^{N}$ is labeled by $x=$ $\left(x_{1}, \ldots, x_{N}\right)=\left(x^{\prime}, y\right)$ where $x^{\prime} \in \mathbb{R}^{N-1}$ and $y=x_{N} \in \mathbb{R}$ (we also use the symbol $\mathbb{R}_{y}$ in place of $\left.\mathbb{R}\right)$. Let $\Omega \subset \mathbb{R}^{N}$ be a measurable set; we denote by $\pi_{N-1}(\Omega)$ the orthogonal projection of $\Omega$ onto $\mathbb{R}^{N-1}$. For all $x^{\prime} \in \pi_{N-1}(\Omega)$, we define

$$
\Omega\left(x^{\prime}\right)=\left\{y \in \mathbb{R}:\left(x^{\prime}, y\right) \in \Omega\right\} .
$$

The Steiner symmetrization of $\Omega$ with respect to the hyperplane $y=0$ is

$$
\Omega^{\sharp}=\left\{\left(x^{\prime}, y\right) \in \mathbb{R}^{N}:-\frac{1}{2}\left|\Omega\left(x^{\prime}\right)\right|_{1}<y<\frac{1}{2}\left|\Omega\left(x^{\prime}\right)\right|_{1}, x^{\prime} \in \pi_{N-1}(\Omega)\right\},
$$

where $\left|\Omega\left(x^{\prime}\right)\right|_{1}$ stands for the Lebesgue measure in $\mathbb{R}_{y}$.

The set $\Omega$ is said to be Steiner symmetric if $\Omega^{\sharp}=\Omega$.

Let $u$ be a nonnegative measurable function defined in $\Omega$; for every $c \in \mathbb{R}$, we denote by $\Omega_{c}=\{x \in \Omega: u(x) \geq c\}$ the level sets of $u$; the Steiner symmetrization $u^{\sharp}$ of $u$ is the function

$$
u^{\sharp}(x)=\sup \left\{c: x \in \Omega_{c}^{\sharp}\right\},
$$

defined in the symmetrized set $\Omega^{\sharp}$. We recall some well-known properties of the Steiner symmetrization:

i) $|\Omega|=\left|\Omega^{\sharp}\right|$;

ii) for every measurable function $\psi$, we have

$$
\int_{\Omega} \psi(u(x)) d x=\int_{\Omega^{\sharp}} \psi\left(u^{\sharp}(x)\right) d x ;
$$

iii) for all $u, v \in L^{2}(\Omega)$, the Hardy-Littlewood's inequality holds:

$$
\int_{\Omega} u(x) v(x) d x \leq \int_{\Omega^{\sharp}} u^{\sharp}(x) v^{\sharp}(x) d x ;
$$


iv) if $u \in W_{0}^{1, p}(\Omega)$, then $u^{\sharp} \in W_{0}^{1, p}\left(\Omega^{\sharp}\right)$ and the Pòlya-Szegö's inequality holds:

$$
\int_{\Omega}|\nabla u(x)|^{p} d x \geq \int_{\Omega^{\sharp}}\left|\nabla u^{\sharp}(x)\right|^{p} d x .
$$

In 3 Brothers and Ziemer have considered the equality case in (2.6), where $\Omega=\mathbb{R}^{N}$ and the Steiner symmetrization is replaced by the decreasing spherical (i.e., Schwarz) symmetrization. They have found sufficient conditions that ensure $u$ is a translate of its symmetrization.

In 7] Cianchi and Fusco have found an analogous result for the Steiner symmetrization. We will use the following result (Theorem 2.6 in [7]) of Cianchi and Fusco.

Theorem 2.2. Let $f: \mathbb{R}^{N} \rightarrow[0, \infty)$ be a strictly convex function vanishing at 0 and satisfying

$$
f\left(x_{1}, \ldots, x_{N-1}, x_{N}\right)=f\left(x_{1}, \ldots, x_{N-1},-x_{N}\right) \quad \forall\left(x_{1}, \ldots, x_{N}\right) \in \mathbb{R}^{N} .
$$

Let $\Omega$ be an open subset of $\mathbb{R}^{N}$ such that $\Omega=\Omega^{\sharp}$ (up to translations along the $y$-axis). Let

$$
W_{0, y}^{1,1}(\Omega)=\left\{u: \Omega \rightarrow \mathbb{R}: u_{0} \in W^{1,1}\left(\omega \times \mathbb{R}_{y}\right) \text { for every open } \omega \subset \subset \pi_{N-1}(\Omega)\right\},
$$

where $u_{0}$ denotes the continuation of $u$ to $\mathbb{R}^{N}$ which vanishes outside $\Omega$. Let $u$ be a nonnegative function from $W_{0, y}^{1,1}(\Omega)$ satisfying

$$
\left|\left\{\left(x^{\prime}, y\right) \in \Omega: \frac{\partial u}{\partial y}\left(x^{\prime}, y\right)=0\right\} \cap\left\{\left(x^{\prime}, y\right) \in \Omega: u\left(x^{\prime}, y\right)<M\left(x^{\prime}\right)\right\}\right|=0,
$$

where $M\left(x^{\prime}\right)=\operatorname{esssup}\left\{u\left(x^{\prime}, y\right): y \in \Omega\left(x^{\prime}\right)\right\}$. If

$$
\int_{\Omega} f\left(\nabla u^{\sharp}\right) d x=\int_{\Omega} f(\nabla u) d x<\infty,
$$

then $u^{\sharp}$ is equivalent to $u$ (up to translations along the $y$-axis).

Remark 2.1. We will use the last theorem with $f(x)=|x|^{p}$ and $u \in W_{0}^{1, p}(\Omega) \subset$ $W_{0, y}^{1,1}(\Omega)$.

We observe that, by Proposition 2.3 in [7], condition (2.7) is equivalent to

$$
\left|\left\{\left(x^{\prime}, y\right) \in \Omega: \frac{\partial u^{\sharp}}{\partial y}\left(x^{\prime}, y\right)=0\right\} \cap\left\{\left(x^{\prime}, y\right) \in \Omega: u^{\sharp}\left(x^{\prime}, y\right)<M\left(x^{\prime}\right)\right\}\right|=0,
$$

which is exactly the counterpart of the hypothesis for Schwarz symmetrization in 3 .

\section{STEINER SYMMETRY}

In 9 the minimization problem (2.2) in a more general case is considered. The set $a d_{\gamma}$ is replaced by a rearrangement class of an arbitrary nonnegative function in $L^{\infty}(\Omega)$. The authors proved a result of existence and characterization of minimizers. Moreover, they have shown uniqueness of the minimizers when $\Omega$ is a ball; more precisely (see Theorem 3.3 in [9]), every minimizer is radially symmetric and decreasing. The first part of their proof relies on Rayleigh's quotient (2.1) and on the Schwarz rearrangement with some related inequalities (analogous to (2.5) and (2.6) $)$. The second part goes through the eigenvalue equation in radial form, 
uses the remarkable result of Brothers and Ziemer in [3] and the characterization (analogous to (2.3)) of minimizers.

Our main result, which is stated in Theorem 3.1, extends the symmetry result of [9] to the case of Steiner symmetry provided the class of rearrengements is $a d_{\gamma}$. Here, we follow the same line of [9] for the first part of the proof. The argument used in 9] in the last part cannot be exploited here because the lack of radial symmetry prevents both the reduction of the equation to the one-dimensional form and the use of 3 .

To overcome these obstructions we use Theorem 2.6 in [7] in place of Theorem 1.1 in [3] and then we proceed with the well-known geometric argument of Serrin (see [21]).

We need the following notation. For a negative real number $a$, we define the horizontal hyperplane

$$
T_{a}=\left\{x \in \mathbb{R}^{N}: y=a\right\},
$$

and the part of $\Omega$ under $T_{a}$

$$
\Omega_{a}=\{x \in \Omega: y<a\} .
$$

For every $x=\left(x^{\prime}, y\right) \in \Omega$, we define its symmetric relative to $T_{a}$

$$
x_{a}=\left(x^{\prime}, 2 a-y\right),
$$

and, for every $x \in \Omega$ such that $x_{a} \in \Omega$ and every function $v$ defined in $\Omega$, let

$$
v_{a}(x)=v\left(x_{a}\right) .
$$

Theorem 3.1. Let $\Omega \subset \mathbb{R}^{\mathbb{N}}$ be a bounded domain of class $C^{2, \sigma}$. Assume $\Omega$ is Steiner symmetric with respect to the hyperplane $T_{0}=\left\{x \in \mathbb{R}^{N}: y=0\right\}$. Then every minimizer $\check{\rho}$ of the problem (2.2) is Steiner symmetric relative to $T_{0}$.

Proof. Recall that by results in [9] there exists a minimizer $\check{\rho}$ of problem (2.2).

We put $\check{\lambda}_{1}=\lambda_{1}(\check{\rho})$ and we denote by $u_{\check{\rho}}$ the corresponding positive first eigenfunction normalized by $\int_{\Omega} \check{\rho} u_{\check{\rho}}^{p} d x=1$.

By Proposition 2.1] the Steiner symmetry of $\check{\rho}$ is a consequence of the Steiner symmetry of $u_{\check{\rho}}$. Hence we need to show that $u_{\check{\rho}}^{\sharp}=u_{\check{\rho}}$.

By (2.1),

$$
\check{\lambda}_{1}=\frac{\int_{\Omega}\left|\nabla u_{\check{\rho}}\right|^{p} d x}{\int_{\Omega} \check{\rho} u_{\check{\rho}}^{p} d x} .
$$

The inequalities (2.5) and (2.6) yield

$$
\int_{\Omega} \check{\rho} u_{\check{\rho}}^{p} d x \leq \int_{\Omega} \check{\rho}^{\sharp}\left(u_{\check{\rho}}^{\sharp}\right)^{p} d x
$$

and

$$
\int_{\Omega}\left|\nabla u_{\check{\rho}}\right|^{p} d x \geq \int_{\Omega}\left|\nabla u_{\check{\rho}}^{\sharp}\right|^{p} d x
$$

Consequently we find

$$
\check{\lambda}_{1}=\frac{\int_{\Omega}\left|\nabla u_{\check{\rho}}\right|^{p} d x}{\int_{\Omega} \check{\rho} u_{\check{\rho}}^{p} d x} \geq \frac{\int_{\Omega}\left|\nabla u_{\check{\rho}}^{\sharp}\right|^{p} d x}{\int_{\Omega} \check{\rho} \sharp\left(u_{\check{\rho}}^{\sharp}\right)^{p} d x} .
$$


Denoting by $u_{\check{\rho}^{\sharp}}$ the normalized positive first eigenfunction corresponding to $\check{\rho}^{\sharp}$, we have

$$
\check{\lambda}_{1} \geq \frac{\int_{\Omega}\left|\nabla u_{\check{\rho}}^{\sharp}\right|^{p} d x}{\int_{\Omega} \check{\rho}^{\sharp}\left(u_{\check{\rho}}^{\sharp}\right)^{p} d x} \geq \frac{\int_{\Omega}\left|\nabla u_{\check{\rho}^{\sharp}}\right|^{p} d x}{\int_{\Omega} \check{\rho}^{\sharp}\left(u_{\check{\rho}^{\sharp}}\right)^{p} d x}=\lambda_{1}\left(\check{\rho}^{\sharp}\right) \geq \check{\lambda}_{1} .
$$

Therefore all the previous inequalities become equalities and yield

$$
\int_{\Omega}\left|\nabla u_{\check{\rho}}\right|^{p} d x=\int_{\Omega}\left|\nabla u_{\check{\rho}}^{\sharp}\right|^{p} d x
$$

and

$$
\int_{\Omega} \check{\rho} u_{\check{\rho}}^{p} d x=\int_{\Omega} \check{\rho}^{\sharp}\left(u_{\check{\rho}}^{\sharp}\right)^{p} d x ;
$$

furthermore, by the simplicity of the first eigenvalue and by (2.4), we conclude that

$$
u_{\check{\rho}}^{\sharp}=u_{\check{\rho}^{\sharp}} .
$$

We set $u_{\tilde{\rho}^{\sharp}}=v$. By (3.1), $v$ satisfies the eigenvalue problem

$$
\begin{cases}-\Delta_{p} v=\check{\lambda}_{1} v^{p-1} \check{\rho}^{\sharp} & \text { in } \Omega, \\ v=0 & \text { on } \partial \Omega,\end{cases}
$$

and $\check{\rho}^{\sharp}$ is a minimizer of (2.2) . Then, by Proposition 2.1, there exists an increasing function

$$
\varphi(t)= \begin{cases}\beta & \text { if } t>l \\ \alpha & \text { if } t \leq l,\end{cases}
$$

where $l$ is a positive constant, such that $\check{\rho}^{\sharp}=\varphi(v)$. Therefore

$$
\begin{cases}-\Delta_{p} v=\check{\lambda}_{1} v^{p-1} \varphi(v) & \text { in } \Omega, \\ v=0 & \text { on } \partial \Omega .\end{cases}
$$

Note that the set $\left\{x \in \Omega: \check{\rho}^{\sharp}=\beta\right\}=\{x \in \Omega: v>l\}$ is open. Now we apply Theorem 2.2 with $f(x)=|x|^{p}$ and $u=u_{\check{\rho}}$. In order to accomplish this step we show that (2.8) holds. In the rest of the proof we will deal with the function $v=u_{\tilde{\rho}^{\sharp}}=u_{\check{\rho}}^{\sharp}$. Actually we will show a stronger condition, i.e.,

$$
\left|\left\{x \in \Omega: \frac{\partial v(x)}{\partial y}=0\right\}\right|=0 .
$$

We introduce the closed sets $Z=\{x \in \Omega: \nabla v=0\}$ and $H=\{x \in \Omega: v=l\}$. Note that the closeness of $Z$ follows by Hopf's Lemma for the $p$-Laplacian operator (see [23]). By Corollary 1.1 in [17] (recall that $v$ is bounded), it follows that $Z$ and $H$ have null measure; therefore, it is enough to show that $\frac{\partial v(x)}{\partial y} \neq 0$ for every $x \in \Omega \backslash(Z \cup H)$. Moreover, since $v$ is Steiner symmetric, it suffices to consider the part of $\Omega \backslash(Z \cup H)$ with negative $y$ component.

Fix a point $x_{0}=\left(x_{0}^{\prime}, b\right)$ in the open set $\Omega \backslash(Z \cup H)$ with $b<0$; then there exists a ball $B=B\left(x_{0}, R\right)$ centered at $x_{0}$ with radius $R<-b$ such that $B \subset \Omega \backslash(Z \cup H)$ and $|\nabla v|,\left|\nabla v_{b}\right| \geq \varepsilon>0$ in $B$ for some positive $\varepsilon$. Moreover, by the connectedness of $B, v$ takes only values either greater than $l$ or less than $l$. Therefore the righthand side of the equation in (3.4) is of class $C^{1}(B)$. By standard regularity theory (see for instance [12] and [15]), $v$ and then $v_{b}$ is of class $C^{2}(B)$. 
The functions $v$ and $v_{b}$ satisfy, respectively, the equations

$$
|\nabla v|^{p-2} \Delta v+(p-2)|\nabla v|^{p-4} \sum_{i, j=1}^{N} v_{x_{i}} v_{x_{j}} v_{x_{i} x_{j}}+\check{\lambda}_{1} v^{p-1} \delta=0 \quad \text { in } B
$$

and (by a reflection through the hyperplane $T_{b}$ )

$$
\left|\nabla v_{b}\right|^{p-2} \Delta v_{b}+(p-2)\left|\nabla v_{b}\right|^{p-4} \sum_{i, j=1}^{N}\left(v_{b}\right)_{x_{i}}\left(v_{b}\right)_{x_{j}}\left(v_{b}\right)_{x_{i} x_{j}}+\check{\lambda}_{1} v_{b}^{p-1} \delta=0 \quad \text { in } B,
$$

for the same constant $\delta$ such that either $\delta=\alpha$ or $\delta=\beta$. Following [21] and since $\check{\lambda}_{1} \delta\left(v_{b}^{p-1}-v^{p-1}\right) \geq 0$ in $\Omega_{b} \cap B$, the function $v_{b}-v$ satisfies

$$
\sum_{i, j=1}^{N} a_{i j}(x)\left(v_{b}-v\right)_{x_{i} x_{j}}+\sum_{i=1}^{N} b_{i}(x)\left(v_{b}-v\right)_{x_{i}} \leq 0 \quad \text { in } \Omega_{b} \cap B,
$$

where

$a_{i j}(x)=\left\{\left|\nabla v_{b}\right|^{p-2}+|\nabla v|^{p-2}\right\} \delta_{i j}+(p-2)\left\{\left|\nabla v_{b}\right|^{p-4}\left(v_{b}\right)_{x_{i}}\left(v_{b}\right)_{x_{j}}+|\nabla v|^{p-4} v_{x_{i}} v_{x_{j}}\right\}$ is uniformly positive definite because $|\nabla v|$ and $\left|\nabla v_{b}\right|$ are both greater than $\varepsilon$ in $B$ and $b_{i}(x)$ are bounded functions.

Therefore we have

$$
\begin{gathered}
\sum_{i, j=1}^{N} a_{i j}(x)\left(v_{b}-v\right)_{x_{i} x_{j}}+\sum_{i=1}^{N} b_{i}(x)\left(v_{b}-v\right)_{x_{i}} \leq 0 \quad \text { in } \Omega_{b} \cap B, \\
v_{b}-v \geq 0 \quad \text { in } \Omega_{b} \cap B \\
v_{b}-v=0 \quad \text { in } \Omega_{b} \cap T_{b} .
\end{gathered}
$$

By the strong maximum principle (see for instance Theorem 3.5 in [12]) the function $v_{b}-v$ is either identically zero or positive in $\Omega_{b} \cap B$. Let us show that the first case is impossible. Suppose $v_{b}(x)=v(x)$ for every $x=\left(x^{\prime}, y\right)$ in $\Omega_{b} \cap B$. Since $v$ is Steiner symmetric we have (recall that $R<-b$ )

$$
v\left(x^{\prime}, b\right) \leq v\left(x^{\prime}, 2 b-y\right)=v\left(x^{\prime}, y\right) \leq v\left(x^{\prime}, b\right)
$$

for all points $\left(x^{\prime}, y\right)$ in $\Omega_{b} \cap B$. Thus

$$
v\left(x^{\prime}, y\right)=v\left(x^{\prime}, b\right)
$$

for all points $\left(x^{\prime}, y\right)$ in $\Omega_{b} \cap B$ and as a consequence

$$
\nabla v\left(x^{\prime}, y\right)=\nabla v\left(x^{\prime}, b\right)
$$

for all points $\left(x^{\prime}, y\right)$ in $\Omega_{b} \cap B$. Now define

$$
\begin{aligned}
& y_{m}=\inf \left\{s \in \mathbb{R}:\left\{x_{0}^{\prime}\right\} \times[s, b) \in \Omega \backslash(Z \cup H)\right. \\
& \text { and equations (3.9) and (3.10) hold for every } \left.\left(x^{\prime}, y\right) \in\left\{x_{0}^{\prime}\right\} \times[s, b)\right\} \text {. }
\end{aligned}
$$

It is clear that $y_{m}$ is well defined and since $v \in C_{\mathrm{loc}}^{1, \tau}(\Omega)$, we have

$$
v\left(x_{0}^{\prime}, y_{m}\right)=v\left(x_{0}^{\prime}, b\right) \neq l
$$

and

$$
\nabla v\left(x_{0}^{\prime}, y_{m}\right)=\nabla v\left(x_{0}^{\prime}, b\right) \neq 0
$$


therefore $\left(x_{0}^{\prime}, y_{m}\right) \in \Omega \backslash(Z \cup H)$. Moreover note that

$$
\frac{\partial v}{\partial y}\left(x_{0}^{\prime}, y_{m}\right)=0 \text {. }
$$

Now we repeat the previous reasoning with $\left(x_{0}^{\prime}, y_{m}\right)$ in place of $\left(x_{0}^{\prime}, b\right)$. Therefore there exists a ball $B^{\prime}=B\left(\left(x_{0}^{\prime}, y_{m}\right), R^{\prime}\right)$ such that the function $v_{y_{m}}-v$ is either identically zero or positive in $\Omega_{y_{m}} \cap B^{\prime}$. In both cases we find a contradiction. In the first case equations (3.9) and (3.10) hold with $b$ and $B$ replaced respectively by $y_{m}$ and $B^{\prime}$ and thus the definition of $y_{m}$ is contradicted.

In the second case $v_{y_{m}}-v$ is a positive function in $\Omega_{y_{m}} \cap B^{\prime}$ and vanishes at the point $\left(x_{0}^{\prime}, y_{m}\right)$. By Hopf's Lemma (see Lemma 3.4 in [12]) applied to the analogous of equation (3.6) for $v_{y_{m}}-v$, we conclude that

$$
-2 \frac{\partial v}{\partial y}\left(x_{0}^{\prime}, y_{m}\right)=\frac{\partial\left(v_{y_{m}}-v\right)}{\partial y}\left(x_{0}^{\prime}, y_{m}\right)<0,
$$

which gives a contradiction with (3.11).

Therefore $v_{b}-v$ is a positive function in $\Omega_{b} \cap B$ and vanishes at the point $\left(x_{0}^{\prime}, b\right)$. By Hopf's Lemma applied to equation (3.6), we conclude that

$$
-2 \frac{\partial v}{\partial y}\left(x_{0}\right)=\frac{\partial\left(v_{b}-v\right)}{\partial y}\left(x_{0}\right)<0 .
$$

This completes the proof.

We conclude the paper with some remarks.

Remark 3.1. From the proof of this theorem it follows that its conclusion still holds when the weight function $\rho$ takes an arbitrary finite number $n$ of positive values. More precisely fix positive constants $\alpha_{1}<\ldots<\alpha_{n}$ and $\gamma_{1}<\ldots<\gamma_{n}$ such that $\gamma_{1}+\cdots+\gamma_{n}=|\Omega|$. Then $a d_{\gamma}$ can be replaced by the collection of all functions of the form

$$
\rho(x)=\sum_{i=1}^{n} \alpha_{i} \chi_{D_{i}}(x),
$$

where $D_{i} \subset \Omega, i=1, \ldots, n$, are arbitrary disjoint measurable sets such that $\left|D_{i}\right|=$ $\gamma_{i}$ and $\Omega=\bigcup_{i=1}^{n} D_{i}$.

Remark 3.2. The problem (2.2) is closely related to the optimization problem considered by Chanillo and others in [4 for $p=2$ and by Pielichowski in 19] for arbitrary $p$. Eigenvalue problem (1.1) is replaced by 1

$$
\begin{cases}-\Delta_{p} \tilde{u}+\tilde{\alpha} \chi_{D}|\tilde{u}|^{p-2} \tilde{u}=\tilde{\lambda}(\tilde{\alpha}, D)|\tilde{u}|^{p-2} \tilde{u} & \text { in } \Omega, \\ \tilde{u}=0 & \text { on } \partial \Omega\end{cases}
$$

where $\tilde{\alpha} \geq 0$ is given and $D$ is a measurable subset of $\Omega$; the measure $|D|=$ $A \in[0,|\Omega|]$ of $D$ is fixed and the first eigenvalue is minimized when $D$ varies in $\Omega$, preserving its measure. A minimizer $D$ is called an optimal configuration. In [19] the author shows that an optimal configuration exists for any choice of the parameters $\tilde{\alpha}$ and $A$; moreover further properties of optimal configurations are proved. An important part of papers [4, [19] and [18] addresses questions about the symmetry of the optimal configurations. In 4 and 18 the Steiner symmetry of the complement of the optimal configurations is shown provided $p=2$ and $\Omega$ is

\footnotetext{
${ }^{1}$ In order to avoid any confusion with our notation, we use $\tilde{u}, \tilde{\alpha}$ and $\tilde{\lambda}$ instead of $u, \alpha$ and $\lambda$.
} 
Steiner symmetric. For arbitrary $p$, Pielichowski (see [19]) proved a radial symmetry result. When $\Omega$ is a ball, the complement of any optimal configuration is still a ball. Of course for $p=2$ this claim follows from the stronger Steiner symmetry result. By Theorem 3.1 we can prove a Steiner symmetry result for Pielichowski's problem. Let $D$ be an optimal configuration with associated parameters $\tilde{\alpha}$ and $A$ such that $\tilde{\alpha}<\bar{\alpha}(A)$ (the quantity $\bar{\alpha}(A)$ is defined in Theorem 2 of [19]) and $\Lambda(\tilde{\alpha}, A)$ the optimal eigenvalue. Then, as a consequence of Theorem 5 in [19], the weight $\check{\rho}=h \chi_{D}+H\left(1-\chi_{D}\right)$ with the constraints $\frac{h}{H}=1-\frac{\tilde{\alpha}}{\Lambda(\tilde{\alpha}, A)}$ and $\gamma=A$ is a minimizer of problem (2.2). Therefore if the hypothesis on $\Omega$ in Theorem 3.1 is satisfied, then $\check{\rho}$ is Steiner symmetric. This implies the Steiner symmetry of the complement of $D$, i.e., we obtain a Steiner symmetry preservation result when $\tilde{\alpha}<\bar{\alpha}(A)$ and $p$ is arbitrary.

\section{REFERENCES}

[1] Aomar Anane, Simplicité et isolation de la première valeur propre du p-laplacien avec poids (French, with English summary), C. R. Acad. Sci. Paris Sér. I Math. 305 (1987), no. 16, 725-728. MR920052 (89e:35124)

[2] G. Barles, Remarks on uniqueness results of the first eigenvalue of the p-Laplacian (English, with French summary), Ann. Fac. Sci. Toulouse Math. (5) 9 (1988), no. 1, 65-75. MR.971814 (90b:35173)

[3] John E. Brothers and William P. Ziemer, Minimal rearrangements of Sobolev functions, J. Reine Angew. Math. 384 (1988), 153-179. MR929981 (89g:26013)

[4] S. Chanillo, D. Grieser, M. Imai, K. Kurata, and I. Ohnishi, Symmetry breaking and other phenomena in the optimization of eigenvalues for composite membranes, Comm. Math. Phys. 214 (2000), no. 2, 315-337, DOI 10.1007/PL00005534. MR1796024(2001i:49077)

[5] S. Chanillo, D. Grieser, and K. Kurata, The free boundary problem in the optimization of composite membranes, Differential geometric methods in the control of partial differential equations (Boulder, CO, 1999), Contemp. Math., vol. 268, Amer. Math. Soc., Providence, RI, 2000, pp. 61-81, DOI 10.1090/conm/268/04308. MR.1804790 (2002a:35207)

[6] Sagun Chanillo, Carlos E. Kenig, and Tung To, Regularity of the minimizers in the composite membrane problem in $\mathbb{R}^{2}$, J. Funct. Anal. 255 (2008), no. 9, 2299-2320, DOI 10.1016/j.jfa.2008.04.015. MR2473259(2010c:49071)

[7] Andrea Cianchi and Nicola Fusco, Steiner symmetric extremals in Pólya-Szegö type inequalities, Adv. Math. 203 (2006), no. 2, 673-728, DOI 10.1016/j.aim.2005.05.007. MR2228056 (2007f:46031)

[8] Steven J. Cox, Extremal eigenvalue problems for the Laplacian, Recent advances in partial differential equations (El Escorial, 1992), RAM Res. Appl. Math., vol. 30, Masson, Paris, 1994, pp. 37-53, DOI 10.2307/25305835. MR1266201 (95f:35182)

[9] Fabrizio Cuccu, Behrouz Emamizadeh, and Giovanni Porru, Optimization of the first eigenvalue in problems involving the p-Laplacian, Proc. Amer. Math. Soc. 137 (2009), no. 5, 1677-1687, DOI 10.1090/S0002-9939-08-09769-4. MR2470826 (2009m:35353)

[10] Mabel Cuesta, Eigenvalue problems for the p-Laplacian with indefinite weights, Electron. J. Differential Equations (2001), No. 33, 9. MR.1836801 (2002b:35165)

[11] François de Thélin, Sur l'espace propre associé à la première valeur propre du pseudolaplacien (French, with English summary), C. R. Acad. Sci. Paris Sér. I Math. 303 (1986), no. 8, 355-358. MR860838 (87i:35147)

[12] David Gilbarg and Neil S. Trudinger, Elliptic partial differential equations of second order, Springer-Verlag, Berlin-New York, 1977. Grundlehren der Mathematischen Wissenschaften, Vol. 224. MR0473443 (57 \#13109)

[13] Antoine Henrot, Extremum problems for eigenvalues of elliptic operators, Frontiers in Mathematics, Birkhäuser Verlag, Basel, 2006. MR2251558(2007h:35242)

[14] Antoine Henrot, Minimization problems for eigenvalues of the Laplacian, J. Evol. Equ. 3 (2003), no. 3, 443-461, DOI 10.1007/s00028-003-0111-0. Dedicated to Philippe Bénilan. MR2019029 (2005a:49078) 
[15] Olga A. Ladyzhenskaya and Nina N. Ural'tseva, Linear and quasilinear elliptic equations, Translated from the Russian by Scripta Technica, Inc. Translation editor: Leon Ehrenpreis, Academic Press, New York-London, 1968. MR0244627 (39 \#5941)

[16] Peter Lindqvist, On the equation $\operatorname{div}\left(|\nabla u|^{p-2} \nabla u\right)+\lambda|u|^{p-2} u=0$, Proc. Amer. Math. Soc. 109 (1990), no. 1, 157-164, DOI 10.2307/2048375. MR1007505 (90h:35088)

[17] Hongwei Lou, On singular sets of local solutions to $p$-Laplace equations, Chin. Ann. Math. Ser. B 29 (2008), no. 5, 521-530, DOI 10.1007/s11401-007-0312-y. MR2447484 (2009j:35099)

[18] Wacław Pielichowski, Some remarks on the optimization of eigenvalue problems involving the p-Laplacian, Opuscula Math. 28 (2008), no. 4, 561-566. MR2480155 (2010d:35257)

[19] Wacław Pielichowski, The optimization of eigenvalue problems involving the p-Laplacian, Univ. Iagel. Acta Math. 42 (2004), 109-122. MR2157626 (2006e:35243)

[20] Shigeru Sakaguchi, Concavity properties of solutions to some degenerate quasilinear elliptic Dirichlet problems, Ann. Scuola Norm. Sup. Pisa Cl. Sci. (4) 14 (1987), no. 3, 403-421 (1988). MR.951227(89h:35133)

[21] James Serrin, A symmetry problem in potential theory, Arch. Rational Mech. Anal. 43 (1971), 304-318. MR0333220 (48 \#11545)

[22] Peter Tolksdorf, Regularity for a more general class of quasilinear elliptic equations, J. Differential Equations 51 (1984), no. 1, 126-150, DOI 10.1016/0022-0396(84)90105-0. MR727034 (85g:35047)

[23] J. L. Vázquez, A strong maximum principle for some quasilinear elliptic equations, Appl. Math. Optim. 12 (1984), no. 3, 191-202, DOI 10.1007/BF01449041. MR768629 (86m:35018)

Mathematics and Computer Science Department, University of Cagliari, via OsPEDALE 72, 09124 CAGLiARI, ItALY

E-mail address: canedda@unica.it

Mathematics and Computer Science Department, University of Cagliari, via OsPEDALE 72, 09124 CAGLIARI, ITALY

E-mail address: fcuccu@unica.it 